\title{
Study on the differences of gene expression between pear and apple wild cultivation materials based on RNA-seq technique
}

Huangwei Zhang ${ }^{1,2+}$, Meng Li ${ }^{1{ }^{*}}$, Min Kong ${ }^{1}$, Jim M. Dunwell ${ }^{3}$, Yuyan Zhang ${ }^{4}$, Chao Yue ${ }^{5}$, Juyou Wu ${ }^{1}$ and Shaoling Zhang ${ }^{1 *}$

\begin{abstract}
Background: Pears and apples are both perennial deciduous trees of the Rosaceae family, and both are important economic fruit trees worldwide. The emergence of many varieties in the market has been mostly domesticated from wild to cultivated and regulated by the differential expression of genes. However, the molecular process and pathways underlying this phenomenon remain unclear. Four typical wild and cultivar pear and apple trees at three developmental stages were used in our study to investigate the molecular process at the transcriptome level.

Result: Physiological observations indicated the obvious differences of size, weight, sugar acid content and peel color in wild and cultivar fruit among each developmental stage. Using next-generation sequencing based RNA-seq expression profiling technology, we produced a transcriptome in procession of a large fraction of annotated pear and apple genes, and provided a molecular basis underlying the phenomenon of wild and cultivar fruit tree differences. 5921 and 5744 differential expression genes were identified in pear and apple at three developmental stages respectively. We performed temporal and spatial differential gene expression profiling in developing fruits. Several key pathways such as signal transduction, photosynthesis, translation and many metabolisms were identified as involved in the differentiation of wild and cultivar fruits.
\end{abstract}

Conclusion: In this study, we reported on the next-generation sequencing study of the temporal and spatial mRNA expression profiling of pear and apple fruit trees. Also, we demonstrated that the integrated analysis of pear and apple transcriptome, which strongly revealed the consistent process of domestication in Rosaceae fruit trees. The results will be great influence to the improvement of cultivar species and the utilization of wild resources.

Keyword: Pear, Apple, Wild cultivar, Differential expression, Domestication, RNA-seq

*Correspondence: mli@njau.edu.cn; slzhang@njau.edu.cn

${ }^{\dagger}$ Huangwei Zhang and Meng Li contributed equally to this work.

${ }^{1}$ College of Horticulture, Nanjing Agricultural University, Nanjing 210095,

China

Full list of author information is available at the end of the article

(c) The Author(s) 2021. Open Access This article is licensed under a Creative Commons Attribution 4.0 International License, which permits use, sharing, adaptation, distribution and reproduction in any medium or format, as long as you give appropriate credit to the original author(s) and the source, provide a link to the Creative Commons licence, and indicate if changes were made. The images or other third party material in this article are included in the article's Creative Commons licence, unless indicated otherwise in a credit line to the material. If material is not included in the article's Creative Commons licence and your intended use is not permitted by statutory regulation or exceeds the permitted use, you will need to obtain permission directly from the copyright holder. To view a copy of this licence, visit http://creativecommons.org/licenses/by/4.0/. The Creative Commons Public Domain Dedication waiver (http://creativeco $\mathrm{mmons}$.org/publicdomain/zero/1.0/) applies to the data made available in this article, unless otherwise stated in a credit line to the data. 


\section{Background}

Pear and apple trees belong to the deciduous perennial trees of Malinae subtribe within Rosaceae (Pyrus) family [1]. Both of them are important economic fruits worldwide, and China is the main producer and consumer (FAO, 2020). Pear originated from the wild pear 'Douli' (P. calleryana Dence) southwest China, which has a cultivation history of more than 3000 years in China [2, 3]. Cultivar pears trees include $P$. bretschneideri, P. pyrifolia, $P$. ussuriensis, $P$. sinkiangensis, and $P$. communis system [4], while typical wild pears include Du pear ( $P$. betulifolia Bunge), Chuan pear (P. pashia Buch), Shan pear ( $P$. ussuriensis Maxim) and Ma pear (P. serrulata Rehd) [5]. Wild apple species originated 3 million years ago, and the fossil remains of apple fruits in the Anatolia Peninsula in Western Asia can be traced back to about 6500 BC [6]. Numbers of studies believed that the wild apple specie in Central Asia was the ancestors of modern cultivated apples, namely $M$. sieversii (Ledeb.) Roem [6, 7].

Fruit tree domestication is a process of adapting wild fruit trees to a new environment by changing their genetic characteristics [8]. Generally, wild species have smaller fruits, oblate in shape, with low sugar content, high acid content, rough pericarp, small leaves and short petiole $[9,10]$. According to individual preferences, people often choose fruit with a large size, bright color and good flavor, all these characteristics are required if a cultivar is to be widely planted. Therefore, the quality of fruit such as fruit size, sugar and acid content, peel color, and fruit firmness has always been a focus of research [1113]. With the completion of several genome sequences in pear and apple [14-17], research on fruit evolution and quality has expanded greatly. Some related genes such as miR172g, MdSWEET9b, MdSWEET15 $a$ and MYB have been identified in fruit trees [7, 18, 19]. Despite a set of draft genome, SNP and QTL in pear and apple [17, 20-22], the molecular process underlying huge changes between wild and cultivar fruit remain unclear.

Measurements of mRNA expression levels, clarity of the regulatory relationships between them are critical to understanding many pathways and biological systems. With the advent of second-generation sequencing-based technologies of RNA-seq, it is possible to measure a genome wide dynamic range of expression in an unbiased manner [23]. RNA-seq technology have a high sensitivity and reproducibility and will undoubtedly lead to novel insights into plant development and response [24].

In the present study, the wild pear 'Douli' ( $P$. calleryana Dence), wild apple 'Xifuhaitang' ( $M$. micromalus Makino) and the cultivar pear 'Dangshansuli' (P. bretschneideri Rehd), cultivar apple 'Golden Delicious' ( $M$. domestica) were selected as typical materials for wild and cultivar fruit tree $[2,14,25,26]$. To comprehensively understand the molecular pathway underlying the huge changes of wild and cultivar at the whole genome level, we have studied the differently expressed genes in different development stages both in pear and apple using RNA-seq technology. The results will further explore the regulation pathways of pear and apple domestication, especially the differences in fruit size, fruit flavor, peel color and fruit resistance, which will be great influence to the improvement of cultivated species and the utilization of wild resources.

\section{Results \\ Transcript RNA sequence dataset of pear and apple libraries}

In this study, RNA of different types of materials (including two species and three developmental stages) were pooled to provide a broad gene library associated with fruit growth and finally sixteen libraries were generated including the fruits and leaves (Fig. 1, Table 1). A total of 14,937,456-30,370,082 reads were obtained from eight libraries of pear, $57.8 \%-71.8 \%$ of which could be mapped to 'Dangshansuli' reference genome and concordantpairs value is $50.5 \%-66.2 \%$ [14]. A total of $16,219,542-$ $34,807,619$ reads were obtained from eight libraries of apple, $61.0 \%-76.3 \%$ of which could be mapped to the 'Golden Delicious' genome and concordant-pairs value is $54.2 \%-72.5 \%$ [17]. We obtained 84,055 transcripts and 40,402 genes with N50 length $2306 \mathrm{bp}$ and median length 1643. 95 bp in pear; we obtained 95,600 transcripts and 56,781 genes with N50 length 2117 bp and median length $1470.5 \mathrm{bp}$ in apple (Table 2).

\section{Physiological index variation at developmental stages of pear and apple fruits}

To better understand the physiological variation among growth, transverse and longitudinal diameter, single fruit weight and sugar acid content from the developmental stages were observed (Fig. 2, Table 3). The transverse and vertical diameter of wild pear at maturity were 1.46 and 1.23 times of that at young fruit stage, respectively (Fig. 2A). The growth rate of wild pear was slow and the fruit size had no obvious change during the fruit development process. The transverse and vertical diameter of cultivar pear at maturity stage were 4.3 and 2.9 times of that at young fruit stage, respectively. The lateral growth rate of cultivar pear is higher than that of longitudinal growth, and the growth rate of fruit size is much faster than that of wild. The transverse and longitudinal diameters of wild apple at the young fruit stage were $12.87 \mathrm{~mm}$ and $13.1 \mathrm{~mm}$ respectively, while at the mature stage they were $25.14 \mathrm{~mm}$ and $23.77 \mathrm{~mm}$, which were about 1.95 and 1.48 times that at young fruit stage (Fig. 2B). The fruit grew slowly and the size did not change 


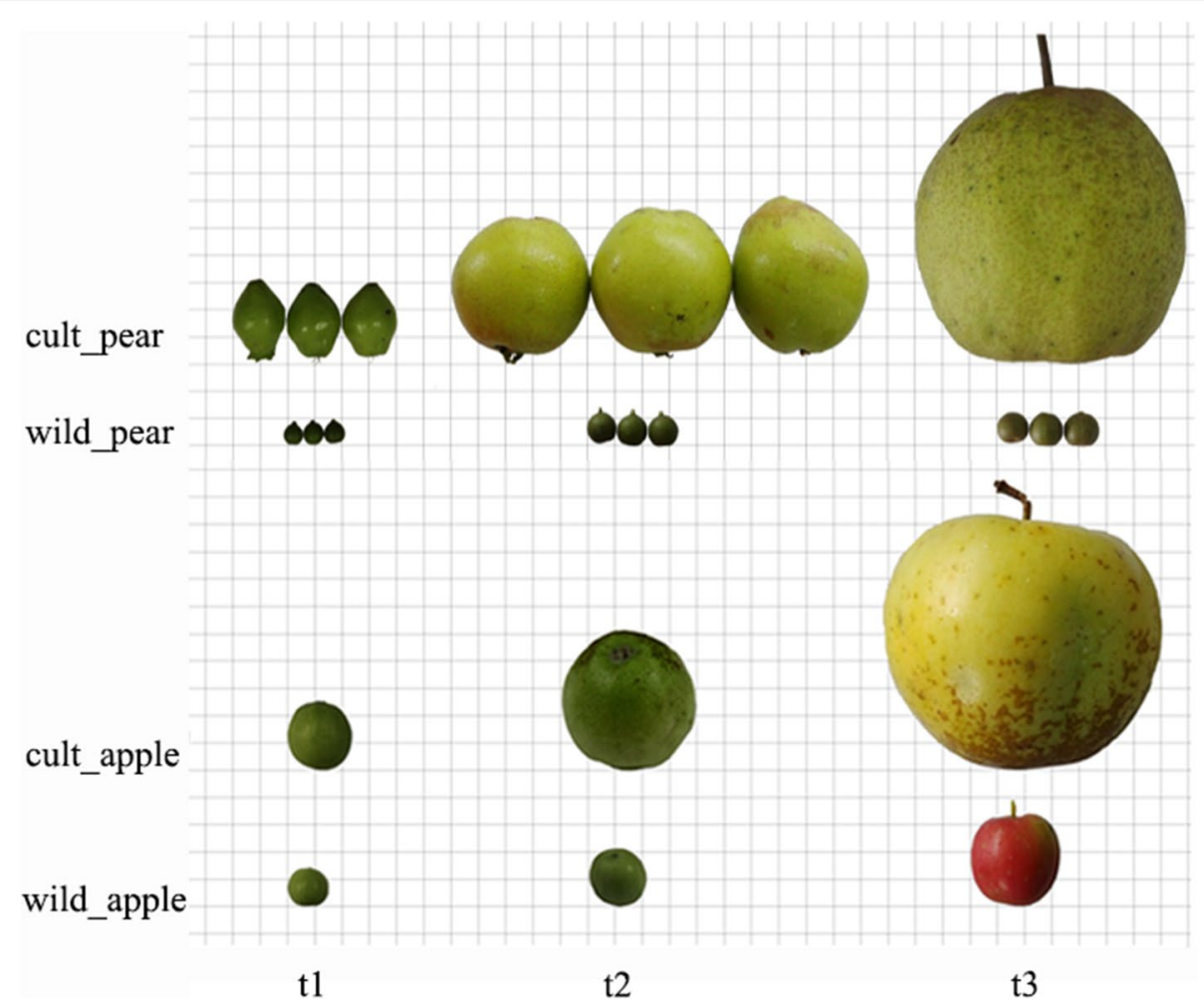

Fig. 1 Plant materials at three developmental stages. $\mathrm{t} 1, \mathrm{t} 2$ and $\mathrm{t} 3$ represent the young fruit stage, the expansion stage and the mature stage respectively. Each square represents $1 \mathrm{~cm}$

significantly. In contrast, the transverse and longitudinal diameters of cultivar apple at maturity were $74.25 \mathrm{~mm}$ and $67.22 \mathrm{~mm}$ respectively, which were about 3.27 and 2.72 times that at the young fruit stage. The weight at maturity stage of cultivar pear is about 447 times of that of wild (Fig. 2C). The weight of cultivar apple at maturity stage was 8.12 times that of wild (Fig. 2D). There are obvious differences in volume and weight between the wild and cultivar, with both fruit characteristics being greater in the cultivar.

The soluble sugars in pear and apple mainly includes sorbitol, fructose, glucose and sucrose [27]. The organic acids are mainly include quinic acid, citric acid, malic acid, oxalic acid and shikimic acid (Table 3) [28]. The total sugar content of cultivar pear at maturity reached $43.6 \mathrm{mg} \bullet \mathrm{g}^{-1}$, and $20.66 \mathrm{mg} \bullet \mathrm{g}^{-1}$ in wild, which showed that the total sugar content of cultivar pear was higher than that of wild. The content of fructose in cultivar pear was $26.24 \mathrm{mg} \bullet \mathrm{g}^{-1}$ at maturity stage, about 13 times that of sucrose. The contents of glucose, fructose and sucrose increased with fruit development, and were positively correlated with total sugar content. There was a negative correlation between sorbitol and total sugar content. The content of citric acid in wild pear is higher than that of other acids. The content of organic acids in wild pear maturity stage is higher than that in cultivar.

Table 1 Stages and abbreviations of the pear and apple libraries

\begin{tabular}{llll}
\hline Pear & & Apple & \\
\hline CP_t1 & May $6^{\text {th }}, 2014 /$ cultivar, young fruit stage fruit & CA_t1 & May $6^{\text {th }}$, 2014/ cultivar, young stage fruit \\
CP_t2 & June $30^{\text {th }}, 2014 /$ cultivar, expansion stage fruit & CA_t2 & June $6^{\text {th }}, 2014 /$ cultivar, expansion stage fruit \\
CP_t3 & September $6^{\text {th }}, 2014 /$ cultivar, mature stage fruit & CA_t3 & September $17^{\text {th }}, 2014 /$ cultivar, mature stage fruit \\
CP_leaf & September $6^{\text {th }}, 2014 /$ cultivar, leaves and branches & CA_leaf & October $30^{\text {th }}, 2014 /$ cultivar, leaves and branches \\
WP_t1 & May $6^{\text {th }}, 2014 /$ wild, young fruit stage fruit & WA_t1 & May $6^{\text {th }}, 2014 /$ wild, young fruit stage fruit \\
WP_t2 & June $30^{\text {th }}, 2014 /$ wild, expansion stage fruit & WA_t2 & June $6^{\text {th }}, 2014 /$ wild, expansion stage fruit \\
WP_t3 & September $6^{\text {th }}, 2014 /$ wild, mature stage fruit & WA_t3 & September $17^{\text {th }}, 2014 /$ wild, mature stage fruit \\
WP_leaf & September $6^{\text {th }}, 2014 /$ wild, leaves and branches & WA_leaf & October $30^{\text {th }}, 2014 /$ wild, leaves and branches \\
\hline
\end{tabular}


Table 2 Summary of mapping reads

\begin{tabular}{llllllll}
\hline Sample id & Total pairs & $\begin{array}{l}\text { Mapped reads } \\
(\%)\end{array}$ & $\begin{array}{l}\text { Concordant pairs } \\
(\mathbf{\%})\end{array}$ & Sample id & Total pairs & $\begin{array}{l}\text { Mapped reads } \\
\text { (\%) }\end{array}$ \\
\hline CP_t1 & $14,937,456$ & 70.6 & 65.8 & CA_t1 & $16,219,542$ & $76.1 \%$ & $71.8 \%$ \\
CP_t2 & $17,645,457$ & 69.3 & 64.2 & CA_t2 & $30,189,007$ & $76.3 \%$ & $72.5 \%$ \\
CP_t3 & $30,034,015$ & 71 & 66.2 & CA_t3 & $34,807,619$ & $72.7 \%$ & $68.7 \%$ \\
CP_leaf & $24,157,305$ & 62.5 & 56.4 & CA_leaf & $22,513,062$ & $72.3 \%$ & $67.9 \%$ \\
WP_t1 & $19,665,537$ & 60.9 & 52.6 & WA_t1 & $17,375,339$ & $63.1 \%$ & $55.7 \%$ \\
WP_t2 & $29,976,758$ & 61.1 & 52.9 & WA_t2 & $29,481,030$ & $64.0 \%$ & $57.4 \%$ \\
WP_t3 & $30,370,082$ & 62.3 & 53.2 & WA_t3 & $30,772,914$ & $65.0 \%$ & $58.4 \%$ \\
WP_leaf & $23,136,777$ & 57.8 & 50.5 & WA_leaf & $25,821,118$ & $61.0 \%$ & $54.2 \%$ \\
\hline
\end{tabular}

The fructose content was higher than other sugars and in cultivar apple at maturity was about 2.6 times of that in wild apple. The total sugar content at maturity of cultivar apple reached $131.23 \mathrm{mg} \bullet \mathrm{g}^{-1}$, and wild apple reached 61.45 $\mathrm{mg} \bullet \mathrm{g}^{-1}$, which showed that the total sugar content in cultivar apple was significantly higher than in wild. The contents of glucose, fructose and sucrose increased during fruit development, and were positively correlated with total sugar content. The content of citric acid and malic acid were higher than the other acids. The highest acid content of wild apple at maturity was citric acid $8.18 \mathrm{mg} \bullet \mathrm{g}^{-1}$, followed by malic acid content of $6.36 \mathrm{mg} \bullet \mathrm{g}^{-1}$. In contrast to sugar content, the

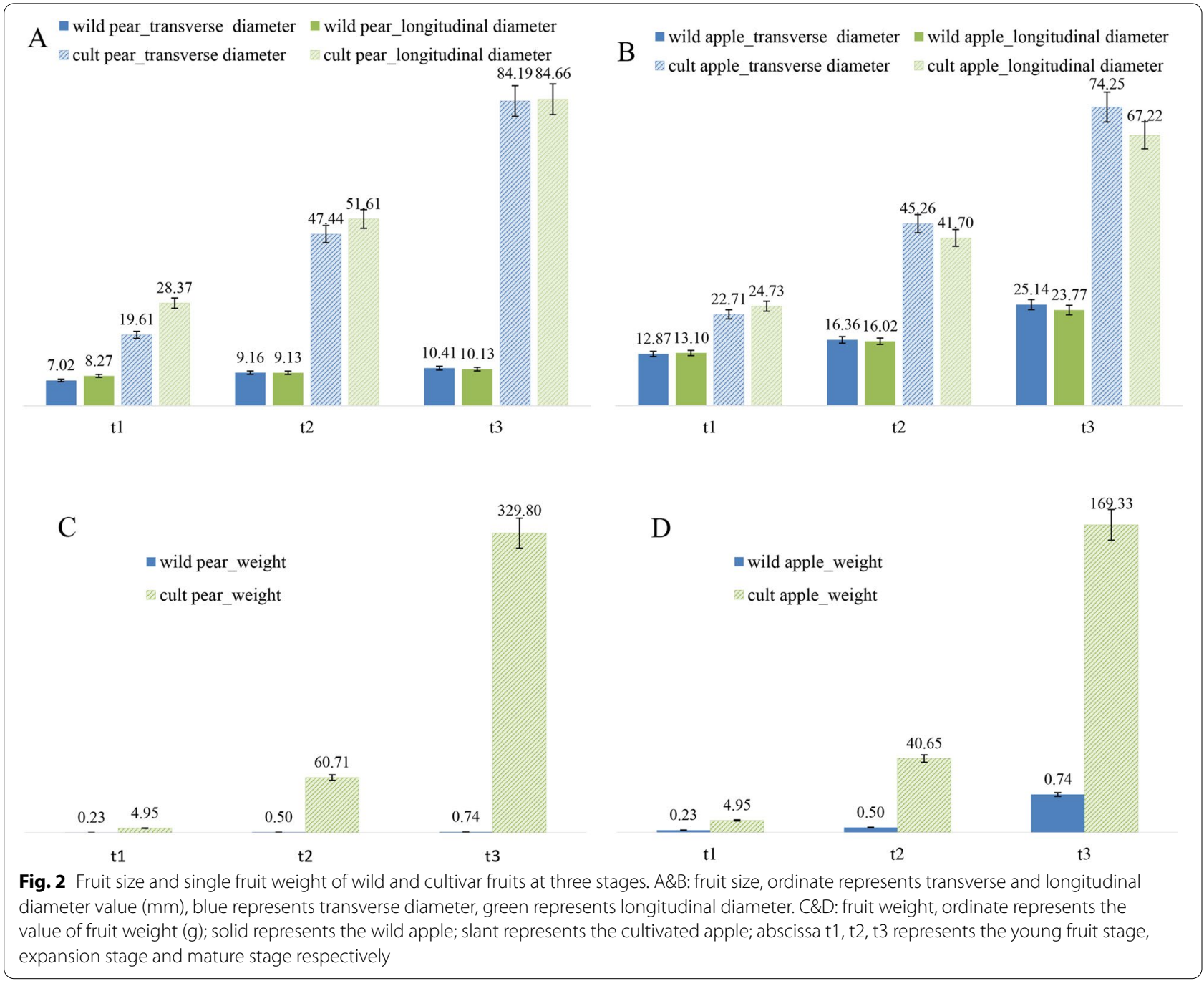


Table 3 Sugar and acid contents of wild and cultivar fruits at three stages

\begin{tabular}{lcccccccccccc}
\hline Content $(\mathbf{m g} / \mathbf{g})$ & CP_t1 & CP_t2 & CP_t3 & WP_t1 & WP_t2 & WP_t3 & CA_t1 & CA_t2 & CA_t3 & WA_t1 & WA_t2 & WA_t3 \\
\hline Sorbitol & 18.20 & 13.09 & 8.67 & 5.88 & 1.35 & 7.02 & 3.77 & 3.47 & 5.11 & 4.73 & 6.27 & 5.83 \\
Fructose & 4.57 & 12.45 & 26.24 & 0.66 & - & 5.27 & 20.09 & 36.72 & 73.27 & 8.24 & 17.48 & 28.67 \\
Glucose & 1.44 & 3.32 & 7.41 & - & - & 4.54 & 7.05 & 13.71 & 27.12 & 4.77 & 12.09 & 14.15 \\
Sucrose & 1.16 & 1.28 & 2.18 & 0.38 & 0.40 & 3.83 & 3.62 & 5.75 & 25.73 & 2.81 & 9.98 & 12.80 \\
Quinic acid & 10.68 & 1.22 & 0.59 & 2.74 & 1.23 & 1.86 & 2.18 & 0.73 & 0.23 & 3.35 & 1.46 & 0.45 \\
Citric acid & - & - & - & - & 0.07 & 4.80 & - & - & - & 7.91 & - & 8.18 \\
Malic acid & 1.47 & 0.96 & 0.84 & 2.69 & 0.90 & 2.59 & 9.62 & 6.66 & 1.97 & 13.87 & 10.91 & 6.36 \\
Oxalic acid & 0.39 & 0.17 & 0.12 & 0.27 & 0.16 & 0.26 & 0.25 & 0.10 & 0.03 & 0.11 & 0.09 & 0.001 \\
Shikimic acid & 0.27 & 0.28 & - & 0.19 & 0.02 & - & 0.05 & 0.03 & - & 0.06 & 0.03 & - \\
\hline
\end{tabular}

organic acid content decreased during fruit maturation. The total acid content in wild was higher than in cultivar apple.

\section{Identification of differentially expressed transcripts and genes in wild and cultivar fruit at three stages}

According to $P<0.001$ and |Fold change $\mid>2$, the significant differential expressed transcripts were identified (Fig. 3). There were 3339, 4005 and 4070 differential expressed transcripts of the two pear varieties at young fruit stage, expanding stage and mature stage, respectively (Fig. 3A). 7051 transcripts were left after removing duplicate transcripts in all three stages, which corresponded to 5921 genes. There are 1228 transcripts that were differentially expressed at all stages; this corresponds to 1068 genes. There were 2261 $(1228+1033)$ differential expressed transcripts existing simultaneously at the expanding stage and the mature stage, and $1699(1228+471)$ and $1631(1228+403)$ at the young fruit stage and expanding stage, the young fruit stage and mature stage, respectively (Fig. 3A).

The numbers of differential expression transcripts were 3188,2975 , and 3918 at apple young fruit stage, expansion stage and mature stage respectively (Fig. 3B). The intersection and union of the three sets were 1065 and
6381 respectively, corresponding to 969 and 5744 genes respectively. The common differentially expressed transcripts between expansion and mature stage, young and expansion stage, young and mature stage were 1586, 1653 and 1526 respectively. The independent differentially expressed transcripts at young fruit stage, expansion stage and mature stage were 1074, 801 and 1871 respectively; these were only expressed in a single stage.

\section{Expression trend analysis of differential expression genes at three periods}

5921 and 5744 different expression genes were further clustered using Short Time-series Expression Miner (STEM) to analyze the expression trend in pear and apple respectively [29]. It identified 16 model expression profiles both in pear and apple (Fig. 4). Colored profiles are statistically significant assigned with $P<0.05$. Among the 7 colored profiles of cultivar pear, profile 13 (397 genes), 15 (239 genes), 12 (208 genes) and 11 (319 genes) were up-regulated, profile 0 (381 genes) and 3 (179 genes) were down-regulated and profile 14 (484 genes) was first up-regulated and then down-regulated (Fig. 4A1). Four
A

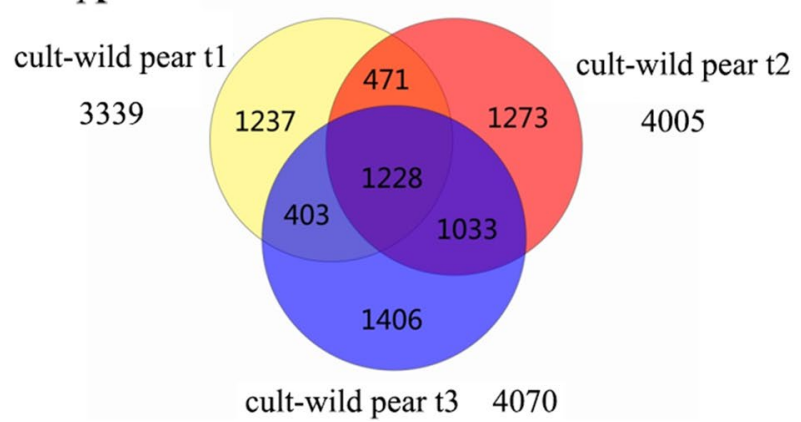

B

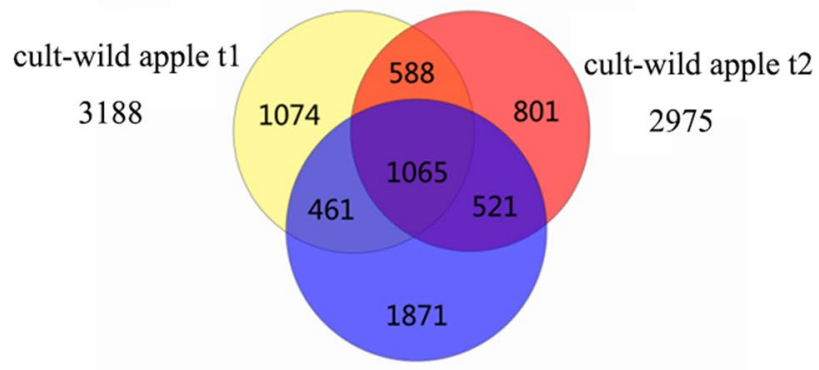

cult-wild apple t3 3918

Fig. 3 The number of differentially expressed transcripts of wild and cultivar samples at three stages. $t 1$, $t 2$ and $t 3$ represent the young fruit stage, the expansion stage and the mature stage respectively 


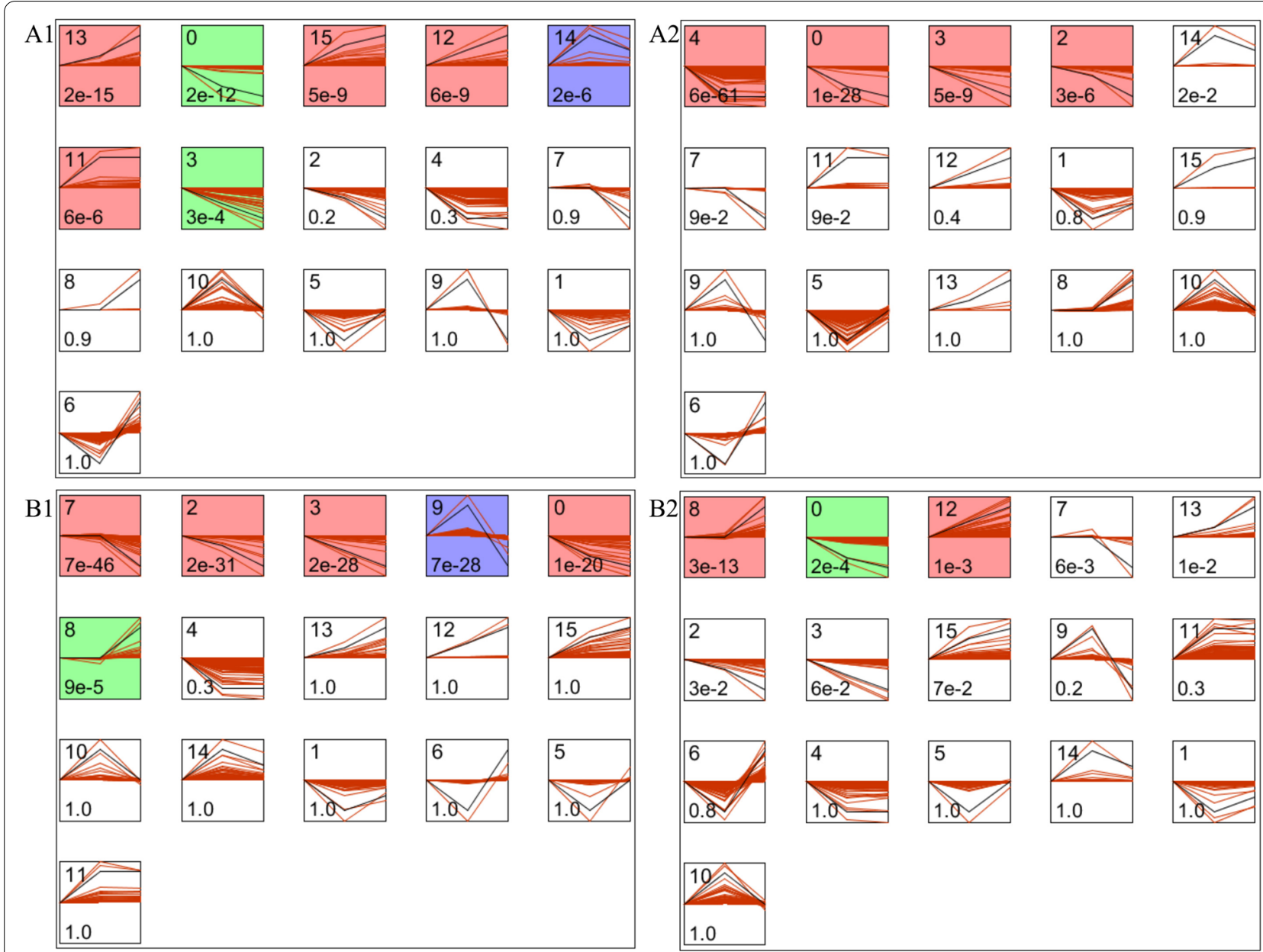

Fig. 4 Expression trend profiles of differentially expressed genes at three stages of cultivar pear (A1), wild pear (A2), cultivar apple (B1) and wild apple (B2) respectively. The number of differential expression genes in pear and apple was 5921, 5744 respectively. Each box represents a different expression profile, colored profiles have a statistically significant number of genes assigned with $P<0.05$, the upper left corner of the digital is profile ID, the lower right corner represents $P$ value

profiles (2007 genes) with significantly colored in wild pear and all of them were down-regulated (Fig. 4A2).

There were 6 colored profiles are significantly at three stages of cultivar apple (Fig. 4B1) and 3 profiles in wild apple (Fig. 4B2). In cultivar apple, profile 7 (539 genes), 2 (345 genes), 3 (298 genes) and 0 (382 genes) were down-regulated, profile 8 (692 genes) was up-regulated, and profile 9 (623 genes) was up-regulated at the beginning and then down-regulated (Fig. 4B1). In wild apple, profile 8 (726 genes) and profile 12 (174 genes) were upregulated, and profile 0 (299 genes) was down-regulated (Fig. 4B2).

\section{Go functional annotation enrichment analysis of differential expression genes}

GO enrichment analysis was carried out on 5921 and 5744 different expression genes in pear and apple respectively, of which 4332 and 4378 genes could be annotated to 3261 and 3190 GO term respectively. GO functional enrichment accorded with a hypergeometric distribution, in which $P<0.05$ was significantly enriched to 855 and 533 terms (Supplementary Fig S1) in pear and apple respectively. GO terms with - $\log (P$-value $)>6$ were further enriched to 36 biological pathways, 16 cell components and 18 molecular functions in pear different expression genes (Fig. 5A), 17 biological pathways, 16 cell components and 6 molecular functions in apple different expression genes (Fig. 5B). The common GO term with -log $(P$-value $)>6$ in pear and apple different expression genes were enriched into 8 biological pathways, 4 cell components and 4 molecular functions (Fig. 5C). The main enriched terms in biological processes were glycolytic process, fatty acid biosynthetic process, response to stress, 


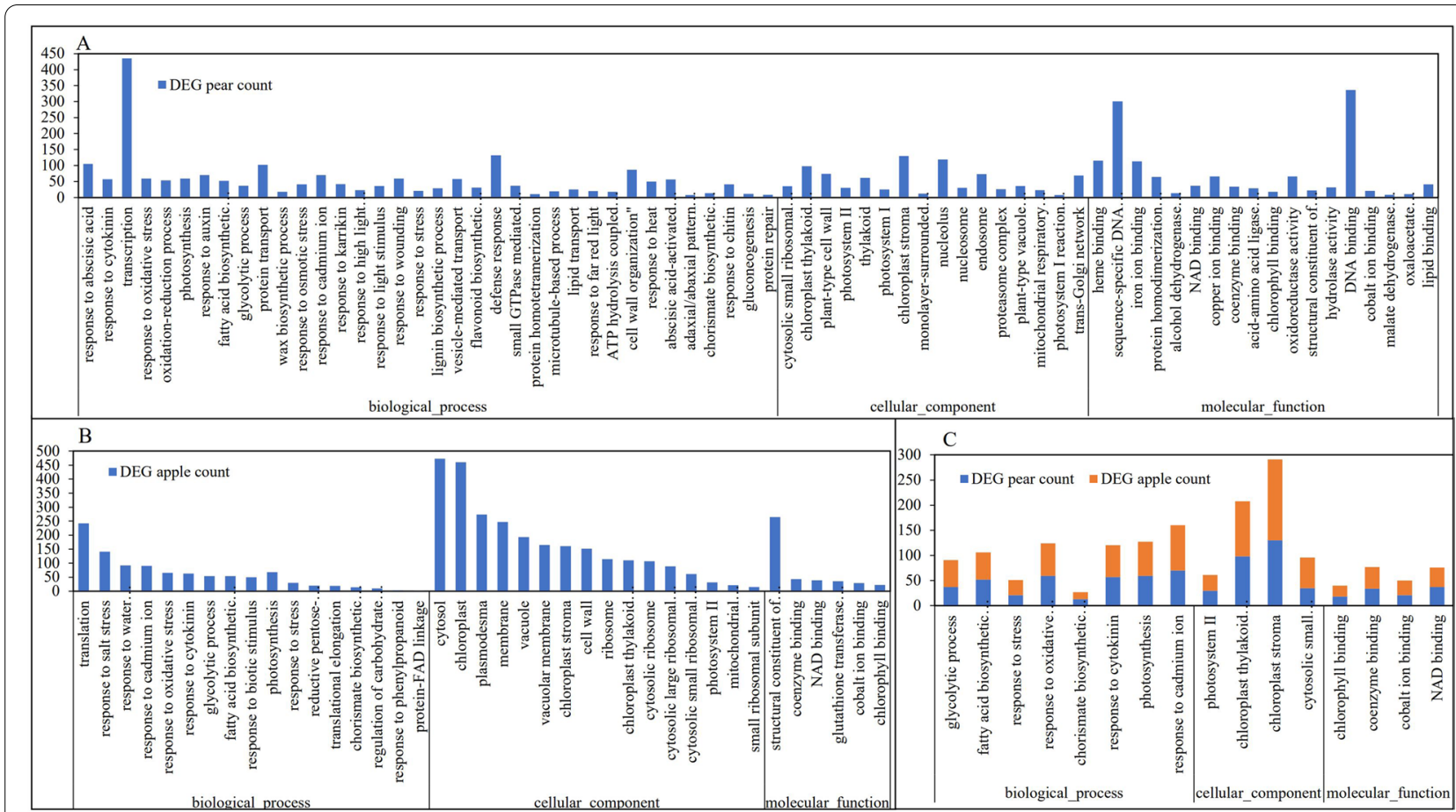

Fig. 5 Functional enrichment of all differentially expressed genes with -log ( $p$-value) $>6.36$ biological pathways, 16 cell components and 18 molecular functions in pear (A), 17 biological pathways, 16 cell components and 6 molecular functions in apple (B). 8 biological pathways, 4 cell components and 4 molecular functions (C) both enriched to pear and apple

response to oxidative stress, chorismate biosynthetic process, response to cytokinin, photosynthesis and response to cadmium ion (Fig. 5C).

\section{Differential expression genes pathway enrichment analysis of pear and apple at three stages}

The Kyoto encyclopedia of genes and genomes (KEGG) pathway can help to further determine biological functions and interactions of genes [30]. Based on a comparison against the KEGG database, of the 40,402 genes in pear, 13,102 (32.43\%) genes had significant matches and were assigned to 133 KEGG pathways; of the 56,781 genes in apple, 18,459 (32.51\%) genes had significant matches and were assigned to 134 KEGG pathways. Among the 5921 different expression genes in pear, 1929 genes were assigned to 123 KEGG pathways, and 50 pathways with $P<0.05$ were significantly enrichment (Supplementary Fig S2). Among the 5744 apple different expression genes, 2578 genes participated in 128 pathways, and 30 pathways with $P<0.05$ were significantly enrichment (Supplementary Fig S3). 24 pathways were significantly enrichment both in pear and apple, which mainly involved in Metabolic pathways (578 genes in pear, 911 genes in apple), Biosynthesis of secondary metabolites (348 genes in pear, 553 genes in apple), Carbon metabolism (85 genes in pear, 161 genes in apple) and Biosynthesis of amino acids (72 genes in pear, 136 genes in apple) etc. (Fig. 6).

\section{Co-expression modules analysis of differential expression genes of pear and apple at three stages}

1068 and 969 genes expressed differently through all development stages in pear and apple respectively. According to the correlation between gene expression, these 1068 and 969 genes been divided into 9 models respectively using WGCNA with Power $=14$ (Fig. 7) [31]. The grey module, which is a non-functional module, has 4 and 2 genes in pear and apple respectively. The correlations between modules and six samples were examined. Most of the 9 modules were negatively correlated with each other in six samples, but the positive correlation was especially significant at certain stages $(P<0.05)$. Function classification indicated that yellow in WP_t1 ( $r=0.97,84$ genes) associated with flavonoid biosynthetic process, response to cadmium ion etc.; red in WP_t3 ( $r=0.90,78$ genes) response to transcription, high light intensity, heat, salt stress and abscisic acid; blue in CP_t1 ( $r=0.90,112$ genes) associated with transcription, glycolytic process, photosynthesis; brown in CP_t2 $(r=0.98,85$ genes) also focuses on response to water deprivation, abscisic acid, cold and salt stress; green in CP_t3 ( $r=0.96,78$ gens) focus on plant-type cell 


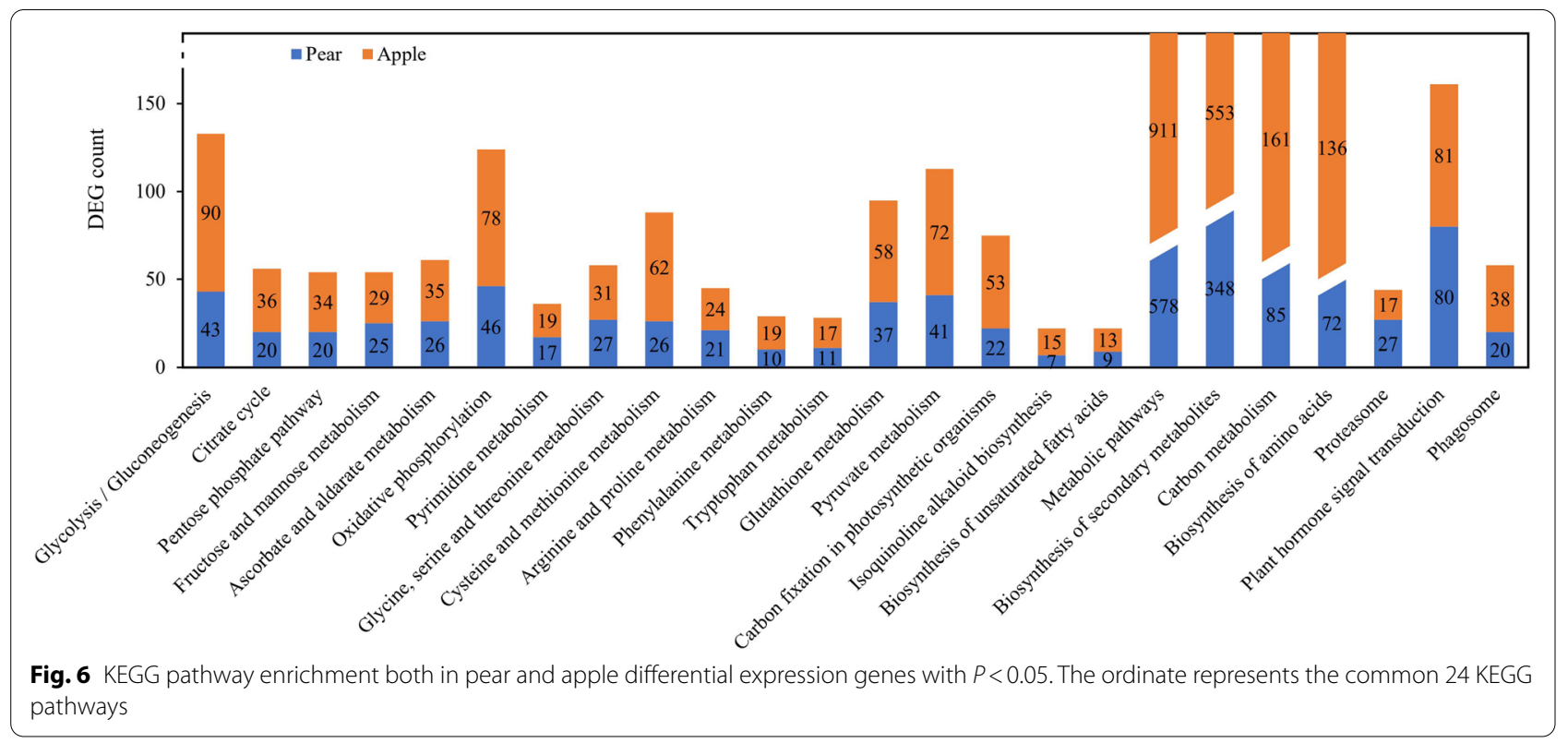

wall organization, malate metabolic process etc. (Fig. 7A, Additional file 1). Module red in WA_t1 $(r=0.93,62$ gens) associated with flavonoid biosynthetic process, anthocyanin-containing compound biosynthetic process, response to stress; brown in WA_t3 $(r=0.93,117$ genes) associated with transcription, defense response, response to cold; pink in CA_t1 ( $r=0.85,35$ genes) associated with response to light stimulus, ATP hydrolysis coupled proton transport; black in CA_t2 $(r=0.86,41$ genes) associated with response to response to water deprivation, photosynthesis, response to cytokinin; yellow in CA_t3 ( $r=0.90,72$ genes) associated with translation, defense response, fatty acid biosynthetic process (Fig. 7B, Additional file 1). Combined with the gene interactions within each module, 10 key genes significantly expressed in each module of pear and apple were selected to conduct the expression level analysis (Additional file 2, Supplementary Fig S4).

\section{Discussion}

In this study, we reported on the next-generation sequencing study of the temporal and spatial mRNA expression profiling of pear and apple fruit trees. A differentially expression genes (DEG) approach with functional classification enabled identifying those mechanisms related to long periods of domestication between wild and cultivated materials [32]. STEM, positive and negative correlation analysis were applied in integrated analysis of DEGs data [29]. WGCNA clusters differential expression genes into modules and correlates them with phenotypes [31]. These provided a molecular basis underlying the differences of wild and cultivated materials.

\section{Reliability of sample data}

Studies have shown that gene expression has biological variability among different individuals, and the variable degree of expression varies among different genes, while transcriptome sequencing technology can not eliminate this variability $[33,34]$. At present, the most common and effective method is to set up biological duplication in experimental design. Although there was no biological duplication for individual specie in this study, we analyzed the correlation of the same gene expression of pear data in this study with published data [14] by comparing the same fruit development stages, and correlation coefficient reached 0.95, 0.96 and $1(P<2.2 \mathrm{e}-16)$ (Supplementary Fig S5). At the same time, the pear and apple species combined as a repeat to jointly analyze the differences between wild and cultivated fruits during domestication which strongly revealed the consistent process of domestication in Rosaceae fruit trees.

\section{Temporal and spatial growth patterns of pear and apple fruits}

The young fruit, expanding and maturity stages are involved in growth phases of fruits and established with phenological period of different area in previous study [14, 35]. To determine transcriptional regulation changes that occurred among wild and cultivar fruits during different growth stages, we analyzed the differential expression genes. In generally, the number of differential expression genes could represent different growth degree, the greater number between species, the larger difference between them [36]. During the growth 


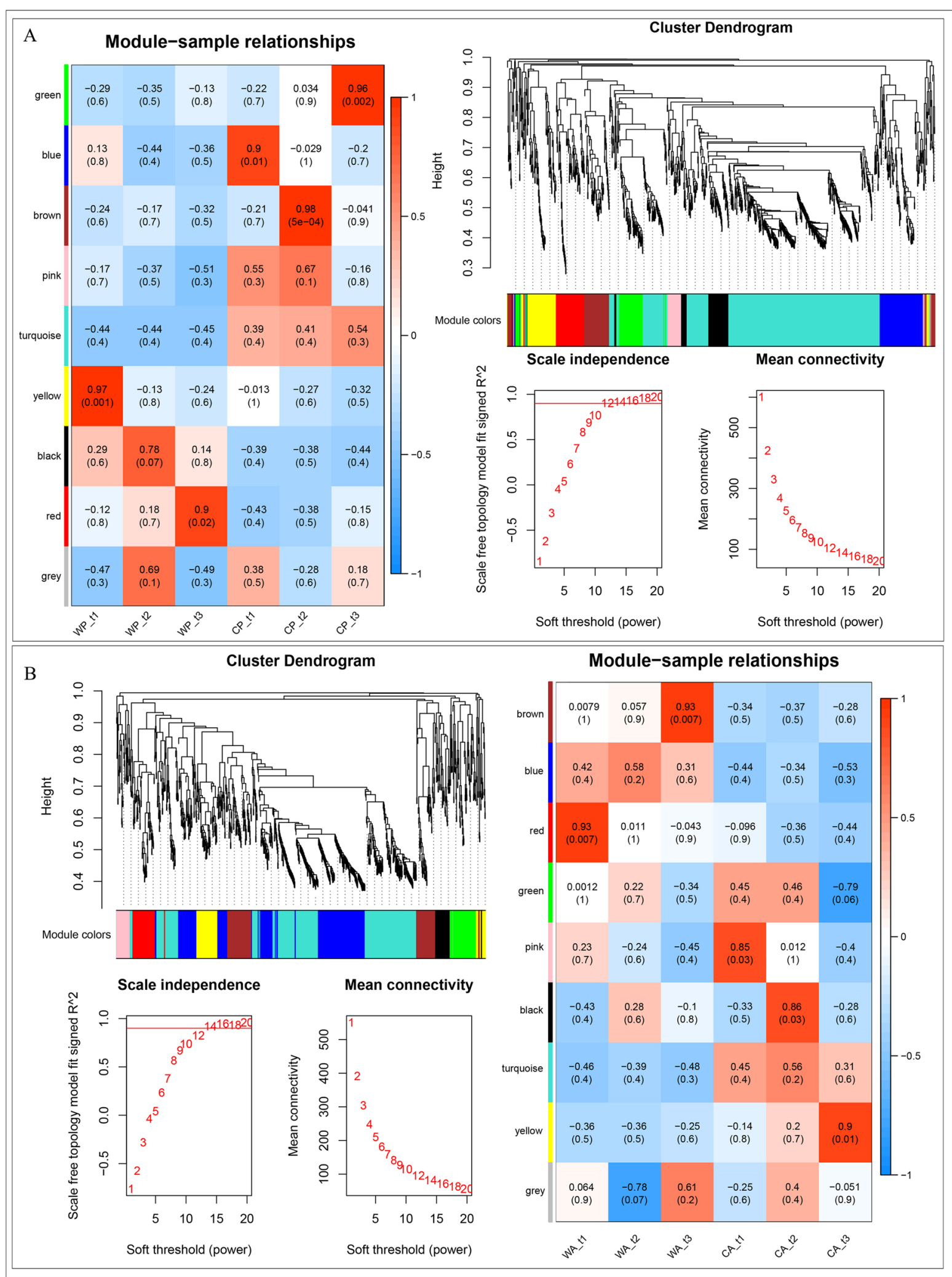

Fig. 7 Co-expression module of 1068 and 969 co-differential expression genes at three stages of pear (A) and apple (B) respectively. Screening of soft-threshold power as 14 at $R^{2}=0.8$ (red line), which divided genes into 9 color modules. Correlation between each module and six samples showed with the correlation coefficient and $P$ value, red indicates positive correlation and blue means negative correlation 
of fruit, the number of differentially expressed genes between wild and cultivated fruit almost become more, and the difference between young fruit stage and maturity stage is the biggest both in pear and apple (Fig. 3). We also found that common differential expression genes were not significantly associated with wild fruit growth at expanding stage both in pear $(r=0.78, P=0.07)$ and apple $(r=0.58, P=0.2)$ (Fig. 7). Compared with others, the number of differential expression genes in wild and cultivar apple at expanding stages was smaller, it may be due to some internal mechanism in the apple that there was little change at this period.

\section{GO and pathways involved in phenotypes and intrinsic qualities of wild and cultivated fruits}

During the long period of domestication from wild fruit to cultivated fruit, the external and internal qualities changed greatly and the data in this study confirm these changes (Figs. 1, 2 and Table 3). The GO and pathways analysis revealed that differential expression genes both in pear and apple involved in glycolytic process, fatty acid biosynthetic process, response to stress, oxidative stress, cytokinin, cadmium ion, photosynthesis, metabolic pathways and signal transduction played major role in domestication of fruits (Figs. 5 and 6).

In this study, fruit size and weight differences were the most obvious feature in the comparison of wild and cultivar materials. Studies have generally believed that they usually determined by the number of cells, cell volume and intercellular space $[15,37]$. Daccord et al. found that in two apple lines, the cell layer which has larger fruit was significantly larger than the another one [15]. Plant endogenous hormones as well as environment factors can regulate progression of plant growth and development through the cell cycle [36]. Several plant hormones, in particular auxin, cytokinin, ethylene and gibberellins are known to regulate cambial development and cell enlargement [38-40]. In the present study, some differential expression genes involved in ethylene biosynthetic process, response to cytokinin, abscisic acid were identified (Additional file 1). This suggests that cell division is essential to rapid volume enlargement of fruits during their early developmental stages.

Photosynthesis is the process by which plants use chlorophyll to convert carbon dioxide and water into organic matter and it plays a key role in plant growth and sugar accumulation [41, 42]. In fact, a common trend both on pear and apple was found that total sugar content of cultivated variety was significantly higher than that of wild and increases during fruit growth, while organic acids showed the opposite trend, these findings are consistent with previous studies [27, 28]. Some studies have suggested that up-regulation of plasma membrane aquaporins improves the photosynthetic activity and growth of trees [43]. Our study also found the cellular component such as photosystem II, ribosomal and molecular function such as chlorophyll binding, coenzyme binding, NAD binding were significantly enriched both in wild cultivar pear and apple differential expression genes (Fig. 5), these results all work together and indicate that photosynthetic capacity has an important effect on the differentiation of wild and cultivated fruit trees. On the other hand, chlorophyll binding and photosynthesis determine the change in peel color [44]. The formation of different colors of fruit is caused by pigments, the pigments in apples are chlorophyll, anthocyanin and carotenoid, which form green, red and yellow respectively [45]. In addition, it was found that differences in light spectrum and intensity had effects on the formation of pigments [46]. In the present study, peel color regulation mainly involved in leucoanthocyanidin reductase activity and flavonoid biosynthetic process, several related genes which have been reported in previous study were up-regulation or down-regulation in apple (Supplementary Fig S6) and they interact to regulate peel color [12, 47, 48].

Some studies reported that wild materials have more advantages in the face of adversity stress or some external condition, and sometimes key genes were selected from them to develop new resistant varieties $[10,49,50]$. Many genes involved in response to stimulus have been reported in the development of rice and sweet orange $[50,51]$. In this study, some differential expression genes involved in defense response to stimulus, such as oxidative stress, cytokinin, auxin or abscisic acid stimulus, cadmium ion, cold, wounding, light were detected during the growth of fruits, indicting the important role of environment factors in fruit's domestication. In addition, there are excellent resistance genes in wild species, which need further study. In the development of domestication from wild to cultivar fruit, the dominant metabolic pathways included biosynthesis of secondary metabolites, carbon metabolism, glycolysis / gluconeogenesis, biosynthesis of amino acids (Fig. 6) have been determined that among the most regulated during developing stages of plants. These metabolic processes can provide the energy and components for DNA replication, translation, signal transduction, hormone biosynthesis and cellular growth [36, 52], which are essential for the rapid domestication of fruits under natural conditions.

\section{Materials and methods}

\section{Plant material and biological measurements}

The wild pear 'Douli' (P. calleryana Decne) and cultivar pear 'Dangshansuli' (P. bretschneideri Rehd) were collected from the experimental farm of Nanjing Agricultural 
University. The wild apple 'Xifuhaitang' ( $M$. micromalus Makino) and cultivar apple 'Golden Delicious' (M. domestica) were collected from Zhenzhou Fruit Research Institute, Chinese Academy of Agricultural Sciences (ZFRI, CAAS). Pear fruits were harvested on May $6^{\text {th }}$, June $30^{\text {th }}$ and September $6^{\text {th }}$ 2014; Apple Fruit were harvested on May $6^{\text {th }}$, June $6^{\text {th }}$ and September $17^{\text {th }} 2014$, and which corresponded to the young fruit stage, expansion stage and maturity stage respectively (Table 1). Ten fruits were picked from each stage. The fruits in the same group were cut into small pieces, pooled and packaged in the field, and immediately frozen in liquid nitrogen and then samples were stored in $-80{ }^{\circ} \mathrm{C}$ until RNA extraction. In addition, the single fruit weight was measured by electronic balance in another ten fruits, the vertical diameter and horizontal diameter of each fruit was measured by vernier caliper on the cross section of the center of the fruit. The measurement of soluble sugar (sorbitol + fructose + glucose + sucrose) and organic acids (quinic acid+citric acid + malic acid+oxalic acid + shikimic acid) was performed by high performance liquid chromatography (HPLC) according to the classical methods as described with slight modification [53]. Five grams of fruit tissue were ground into powder with liquid nitrogen in a mortar and pestle. After mixed with $6 \mathrm{ml} 80 \%$ ethanol and placed into a $37{ }^{\circ} \mathrm{C}$ bath for $30 \mathrm{~min}$. The mixture was centrifuged at $15,000 \mathrm{~g}$ for $15 \mathrm{~min}$ at $4{ }^{\circ} \mathrm{C}$. The above steps were repeated three times to make sure all sugars and acids were extracted for a total volume of supernatants of $50 \mathrm{~mL}$. The supernatant was recovered and immediately filtered through a SEP-C18 column (Waters, WAT020515) to eliminate any interfering apolar residues and through a $0.45 \mu \mathrm{m}$ Sep-Pak filter (Jasco France, TR200102) to eliminate large particles. The extract was then ready for HPLC system of sugar and acid contents measurement following the characteristics described by Yao et al. (2010) and Sha et al. (2011) respectively [27, 28]. Sample contents were established using external standards and expressed in milligram per gram fresh weight (FW).

\section{Library preparation for transcriptome sequencing}

The fruit tissues from 12 samples (three development stages, four individuals) were collected for RNA preparation. Total RNA was extracted using a TRIzol reagent (Gibco BRL) following the manufacturer's instructions and mRNA was purified from total RNA using poly-T oligoattached magnetic beads. RNA concentration and purity were measured using NanoDrop 2000 (Thermo Fisher Scientific, Wilmington, DE). After fragmentation, first strand cDNA was synthesized using random hexamer primer and second strand cDNA synthesis was subsequently performed using DNA Polymerase I and RNase H. In order to select cDNA fragments of preferentially $240 \mathrm{bp}$ in length, the library fragments were purified with AMPure XP system
(Beckman Coulter, Beverly, USA). After PCR amplification of the selected fragment, library quality was assessed on the Agilent Bioanalyzer 2100 system and then sequenced with Illumina platform and paired-end reads were generated. The raw data of the experiment are submitted to Sequence Read Archive (SRA) of NCBI under accession number SRR9291270, SRR9291271, SRR9891658 and SRR9891659.

\section{Transcriptome data analysis}

Raw reads of fastq format were firstly processed through in-house perl scripts. In this step, the adaptor sequences and low-quality sequence reads were removed from the data sets. Raw sequences were transformed into clean reads after data processing. These clean reads were then mapped to the pear and apple reference genome sequence respectively by Tophat tool soft (http://ccb.jhu. edu/software/tophat/index.shtml) [14, 17]. Only reads with a perfect match or one mismatch were further analyzed and annotated based on the reference genome. Gene function was annotated based on the following databases: $\mathrm{Nr}$ (NCBI non-redundant protein sequences); Nt (NCBI non-redundant nucleotide sequences); Pfam (Protein family); KOG/COG (Clusters of Orthologous Groups of proteins); Swiss-Prot (A manually annotated and reviewed protein sequence database); KO (KEGG Ortholog database); GO (Gene Ontology). Gene expression levels were estimated by transcripts per million (TPM) using Kallisto software (https://pachterlab.github. io/kallisto/).

\section{Differential expression analysis}

The differential expression transcripts and genes in fruits were analyzed by R-package DEGseq [32]. DEGseq is more efficient in the detection of differentially expressed genes directly by using the number of reading segments from transcripts, and in the detection of the MA map of differentially expressed genes by mapping the $\log _{2}$ value of reading segments from a particular transcript. It is assumed that the number of reads to a specific gene in two different samples is $\mathrm{C}_{1}$ and $\mathrm{C}_{2}$ respectively: $\mathrm{M}=\log _{2} \mathrm{C}_{1} \quad-\quad \log _{2} \mathrm{C}_{2}, \quad \mathrm{~A}=\left(\log _{2} \mathrm{C}_{1}+\log _{2} \mathrm{C}_{2}\right) / 2 . \quad P$ value $<0.001$ and $\mid$ Fold change $\mid>2$ was set as the threshold for significantly differential expression.

\section{GO and KEGG pathway enrichment analysis of differential expression genes}

Gene Ontology (GO) is an international web site for functional annotations of genes, providing a range of semantics for describing the characteristics of genes and gene products, including cellular components, molecular functions and biological pathways (http://www. geneontology.org/). Kyoto Encyclopedia of Genes and Genomes (KEGG) a database resource for understanding 
high-level functions and utilities of the biological system, such as the cell, the organism and the ecosystem, from molecular-level information, especially large-scale molecular datasets generated by genome sequencing and other high-throughput experimental technologies (http://www.kegg.jp/). Gene Ontology and KEGG pathway enrichment analysis of the differential expression genes were implemented by $\mathrm{R}$ script based non-central hyper-geometric distribution with $P$ value $<0.05$ [54].

\section{Short time series expression analysis of differential expression genes}

Short Time-series Expression Miner (STEM) [29] is a software for sequence clustering, comparison and visual expression in a short time. It is suitable for 3-8 time points. The maximum number of model profiles is 50 , the maximum unit change in model profiles between time points is 2. STEM can order profiles by 'Swap rows and columns', 'Profile ID', 'Significance' and 'Correlation'. In this study, all differential expression genes of four individuals (wild and cultivar pear, wild and cultivar apple) as input file, and regulation trend significantly changed with $P$ value $<0.05$.

\section{Differential expression genes co-expression module recognition}

The R package of WGCNA (Weighted Gene Co-Expression Network Analysis) is a common tool for weighted correlation network analysis [31]. In this study, 1068 and 969 common differential expression genes at three stages in pear and apple as input data respectively. All data were complete and no outliers. The soft threshold power was screened and the gene was divided into nine modules by selecting power as 14 . Modules are defined as clusters of densely interconnected genes. Finally, the correlation among each modules and phenotypes at three development stages were calculated, the significantly correlated modules with $r>0.8$ and $P$ value $<0.05$.

\section{Supplementary Information}

The online version contains supplementary material available at https://doi. org/10.1186/s12870-021-03051-0.

\section{Additional file 1.}

Additional file 2.

Additional file 3 .

Acknowledgements

Not applicable.

\section{Authors' contributions}

HWZ analyzed the data and drafted the manuscripts. ML designed experiment, collected samples and revised the manuscript. MK, JMD, YYZ and CY revised the manuscript and provided guidance for article writing. JYW and SLZ supported for experimental design and revised the manuscript. All authors have read and approved the manuscript.

Funding

This project was supported by the Fundamental Research Funds for the Central Universities, No. KYYJ202117.

Availability of data and materials

Raw reads of the experiment are submitted to NCBI SRA database (https:// www.ncbi.nlm.nih.gov/sra), the accession number of the study is SRR9291270, SRR9291271, SRR9891658 and SRR9891659.

\section{Declarations}

Ethics approval and consent to participate

Not applicable.

\section{Consent for publication}

Not applicable.

\section{Competing interests}

The authors declare that they have no competing interests.

\section{Author details}

${ }^{1}$ College of Horticulture, Nanjing Agricultural University, Nanjing 210095, China. ${ }^{2}$ College of Agro-Grassland Science, Nanjing Agricultural University, Nanjing 210095, China. ${ }^{3}$ School of Agriculture, Policy and Development, University of Reading, Earley Gate, Reading, UK. ${ }^{4}$ Institute of Pomology, Jiangsu Academy of Agricultural Sciences/Jiangsu Key Laboratory of Horticultural Crop Genetic Improvement, Nanjing 210014, Jiangsu, China. ${ }^{5}$ China Tobacco Jiangsu Industrial Co., Ltd, Nanjing 210019, China.

Received: 27 January 2021 Accepted: 11 May 2021

Published online: 04 June 2021

\section{References}

1. Potter D, Eriksson T, Evans RC, Oh S, Smedmark JEE, Morgan DR, Kerr M, Robertson KR, Arsenault M, Dickinson TA. Phylogeny and classification of Rosaceae. Plant Syst Evol. 2007;266(1-2):5-43.

2. Jang JT. Identification of Pyrus species by leaf peroxidase isozyme phenotypes. J Jpn Soc Horticult Sci. 1992;61(2):273-86.

3. Silva GJ, Souza TM, Barbieri RL, Oliveira. Origin, domestication, and dispersing of pear (Pyrus spp.). Adv Agric. 2014:2014(1):1-8.

4. Zheng X, Cai D, Yao L, Teng Y. Non-concerted ITS evolution, early origin and phylogenetic utility of ITS pseudogenes in Pyrus. Mol Phylogenet Evol. 2008;48(3):892-903.

5. Tamura F, Tanabe K, Itai A, Morimoto M. Variations in the chilling requirements for breaking leaf bud endodormancy in wild pear species and pear cultivars. J Jpn Soc Horticult Sci. 2008;70(5):596-8.

6. Vavilov NI. Studies on the origin of cultivated plants. Trudy Byuro Prikl Bot. 1926;16:139-245.

7. Duan N, Yang B, Sun H, Nan W, Chen X. Genome re-sequencing reveals the history of apple and supports a two-stage model for fruit enlargement. Nat Commun. 2017:8(1):249.

8. Pinhas. Domestication of fruit trees. Dev Agric. 1986;16:201

9. Voltas J, Pemán J, Fusté F. Phenotypic diversity and delimitation between wild and cultivated forms of the genus Pyrus in North-eastern Spain based on morphometric analyses. Genet Resour Crop Evol. 2007;54(7):1473-87.

10. Li WM, Dita M, Rouard M, Wu W, Ge XJ. Deep RNA-seq analysis reveals key responding aspects of wild banana relative resistance to Fusarium oxysporum f. sp. cubense tropical race 4. Funct Integr Genomics. 2020;20:551-62.

11. Li M, Li L, Dunwell JM, Qiao X, Liu X, Zhang S. Characterization of the lipoxygenase (LOX) gene family in the Chinese white pear ( Pyrus bretschneideri ) and comparison with other members of the Rosaceae. BMC Genomics. 2014;15(1):444 
12. Zhang XJ, Wang $L X$, Liu YL, Chen XX, Yang YZ, Zhao ZY. Differential gene expression analysis of 'Granny Smith' apple (Malus domestica Borkh.) during fruit skin coloration. S Afr J Bot. 2013;88:125-31.

13. Li M, Li P, Ma F, Dandekar AM, Cheng L. Sugar metabolism and accumulation in the fruit of transgenic apple trees with decreased sorbitol synthesis. Horticult Res. 2018;5(1):1.

14. Wu J, Wang Z, Shi Z, Zhang S, Ming R, Zhu S, Khan MA, Tao S, Korban SS, Wang $\mathrm{H}$. The genome of the pear (Pyrus bretschneideri Rehd.). Genome Res. 2013:23(2):396-408.

15. Daccord N, Celton JM, Linsmith G, Becker C, Choisne N, Schijlen E, Henri V, Bianco L, Micheletti D, Velasco R. High-quality de novo assembly of the apple genome and methylome dynamics of early fruit development. Nat Genet. 2017;49(7):1099

16. Zhang L, Hu J, Han X, Li J, Gao Y, Richards CM, Zhang C, Tian Y, Liu G, Gul $H$. A high-quality apple genome assembly reveals the association of a retrotransposon and red fruit colour. Nat Commun. 2019;10(1):1494.

17. Velasco R, Zharkikh A, Affourtit J, Dhingra A, Cestaro A, Kalyanaraman A, Fontana P, Bhatnagar SK, Troggio M, Genetics DP. The genome of the domesticated apple (Malus $\times$ domestica Borkh.). Nat Genet. 2010:42(10):833-9.

18. Zhen Q, Fang T, Peng Q, Liao L, Zhao L, Owiti A, Han Y. Developing genetagged molecular markers for evaluation of genetic association of apple SWEET genes with fruit sugar accumulation. Horticult Res. 2018;5(1):14.

19. Vimolmangkang S, Han Y, Wei G, Korban SS. An apple MYB transcription factor, MdMYB3, is involved in regulation of anthocyanin biosynthesis and flower development. BMC Plant Biol. 2013;13(1):176.

20. Chagnã D, Crowhurst RN, Pindo M, Thrimawithana A, Deng C, Ireland H, Fiers M, Dzierzon H, Cestaro A, Fontana P. The draft genome sequence of European pear (Pyrus communis L. 'Bartlett'). Plos One. 2014;9(4):e92644.

21. Wu J, Li LT, Li M, Khan MA, Li XG, Chen H, Yin H, Zhang SL. High-density genetic linkage map construction and identification of fruit-related QTLS in pear using SNP and SSR markers. J Exp Bot. 2014;65(20):5771-81.

22. Potts SM, Khan MA, Han Y, Kushad MM, Korban SS. Identification of Quantitative Trait Loci (QTLs) for Fruit Quality Traits in Apple. Plant Mol Biol Rep. 2013;32(1):109-16.

23. Wang Z, Gerstein M, Snyder M. RNA-Seq: a revolutionary tool for transcriptomics. Nat Rev Genet. 2009;10(1):57.

24. Haas BJ, Zody MC. Advancing RNA-Seq analysis. Nat Biotechnol. 2010;28:421-3.

25. Zhen Z, Sun A, Yu C, Sheng B, Cheng YJ. Agrobacterium-mediated transformation of the apple rootstock Malus micromalus Makino with the Rolc gene. Vitro Cell Dev Biol Plant. 2006;42(6):491-7.

26. Li X, Ling K, Zhang J, Xie Y, Wang L, Yan Y, Na W, Xu J, Li C, Wang W. Improved hybrid de novo genome assembly of domesticated apple ( Malus x domestica ). Gigascience. 2016;5(1):1-5.

27. Yao GF, Zhang SL, Cao YF, Liu J, Wu J, Yuan J, Zhang HP, Xiao CC. Characteristics of components and contents of soluble sugars in pear fruits from different species. Scientia Agricultura Sinica. 2010;43(20):4229-37.

28. Sha S, Li J, Wu J, Zhang S. Characteristics of organic acids in the fruit of different pear species. Afr J Agric Res. 2011;6(10):2403.

29. Ernst J, Barjoseph Z. STEM: a tool for the analysis of short time series gene expression data. BMC Bioinformatics. 2006;7(1):191.

30. Minoru K, Michihiro A, Susumu G, Masahiro H, Mika H, Masumi I, Toshiaki K, Shuichi K, Shujiro O, Toshiaki TJ. KEGG for linking genomes to life and the environment. Nucleic Acids Res. 2008:36(Database issue):480-4.

31. Peter $L$, Steve H. WGCNA: an R package for weighted correlation network analysis. BMC Bioinformatics. 2008;9:559.

32. Wang L, Feng Z, Wang X, Wang X, Zhang XJ. DEGseq: an R package for identifying differentially expressed genes from RNA-seq data. Bioinformatics. 2010:26(1):136-8.

33. Hansen KD, Wu Z, Irizarry RA, Leek JT. Sequencing technology does not eliminate biological variability. Nat Biotechnol. 2012;29(7):572-3.

34. Elowitz MB, Levine AJ, Siggia ED, Swain PS. Stochastic gene expression in a single cell. Science. 2002:9:297.

35. Zhang $X$, Koo J, Eun JB. Antioxidant Activities of Methanol Extracts and phenolic compounds in Asian pear at different stages of maturity. Food Sci Biotechnol. 2006;15(1):44-50.
36. He CY, Cui K, Zhang JG, Duan AG, Zeng YF. Next-generation sequencingbased mRNA and microRNA expression profiling analysis revealed pathways involved in the rapid growth of developing culms in Moso bamboo. BMC Plant Biol. 2013;13(1):119

37. Goffinet MC, Robinson TL, Lakso AN. A comparison of "Empire" apple fruit size and anatomy in unthinned and hand-thinned trees. J Pomology Horticult Sci. 1995:70(3):375-87.

38. Liao MA, Liu X, Deng GT, Chen SB, Ren YJ, Liu WG. Studies on the changes of endogenous hormones during fruit growth and development of two pear cultivars. J Fruit Sci. 2009;26:25-31.

39. Teale WD, Paponov IA, Palme K. Auxin in action: signalling, transport and the control of plant growth and development. Nat Rev Mol Cell Biol. 2006;7(11):847-59.

40. Love J, Bioerklund S, Vahala J, Hertzberg M, Kangasjaervi J, Sundberg B. Ethylene is an endogenous stimulator of cell division in the cambial meristem of Populus. Proc Natl Acad Sci USA. 2009;106(14):5984-9.

41. Zelitch I. Photosynthesis, photorespiration and plant productivity. Q Rev Biol. 1971;301-37.

42. Cha-Um S, Charoenpanich A, Roytrakul S, Kirdmanee C. Sugar accumulation, photosynthesis and growth of two indica rice varieties in response to salt stress. Acta Physiol Plant. 2009;31(3):477-86.

43. Tsuchihira A, Hanba YT, Kato N, Doi T, Maeshima M. Effect of overexpression of radish plasma membrane aquaporins on water-use efficiency, photosynthesis and growth of Eucalyptus trees. Tree Physiol. 2010;30(3):417-30

44. Hetherington Suzan EJ. Profiling photosyrithetic competence in mango fruit. J Pomology Horticult Sci. 2015;72(5):755-63.

45. Karanjalker GR, Ravishankar KV, Shivashankara KS, Dinesh MR, Roy TK, Sudhakar Rao DV. A study on the expression of genes involved in Carotenoids and Anthocyanins during ripening in fruit peel of green, yellow, and red colored mango cultivars. Appl Biochem Biotechnol. 2018;184:140.

46. Pearce GW, Streeter LR. A report on the effect of light on pigment formation in apples. J Biol Chem. 1931;92:743-9.

47. Ban Y, Honda C, Hatsuyama Y, Igarashi M, Bessho H, Moriguchi T. Isolation and functional analysis of a MYB transcription factor gene that is a key regulator for the development of red coloration in apple skin. Plant Cell Physiology. 2007:48(7):958.

48. Takos AM, Felix WJ, Jacob SR, Jochen B, Robinson SP, Walker AR. Lightinduced expression of a MYB gene regulates anthocyanin biosynthesis in red apples. Plant Physiol. 2018;142(3):1216-32.

49. Gunasekera D, Santakumari M, Glinka Z, Berkowitz GA. Wild and cultivated barley genotypes demonstrate varying ability to acclimate to plant water deficits. Plant Sci. 1994;99(2):125-34

50. Jiang W. Screening and utilization of wild rice with blast resistance genes. Entia Agricutura Sinica. 1996;29(6):21-28.

51. Qiang X, Liu Y, Zhu A, Wu X, Ye J, Yu K, Guo W, Deng X. Discovery and comparative profiling of microRNAs in a sweet orange red-flesh mutant and its wild type. BMC Genomics. 2010;11(1):246.

52. Li Kun, Wang Yongzhang, Qu Haiyong. RNA-Seq analysis of compatible and incompatible styles of Pyrus species at the beginning of pollination. Plant Mol Biol. 2020;102(3):287-306.

53. Tikhomirov MM, Khorlin AY, Voelter W, Bauer H. High-performance liquid chromatographic investigation of the amino acid, amino sugar and neutral sugar content in glycoproteins. J Chromatogr A. 1978;167(DEC):197-203.

54. Young M, Wakefield MJ, Smyth GK, Oshlack A. Gene ontology analysis for RNA-seq: accounting for selection bias. Genome Biol. 2010;11(2):R14-R14.

\section{Publisher's Note}

Springer Nature remains neutral with regard to jurisdictional claims in published maps and institutional affiliations. 